\title{
Localization of nuclear retained mRNAs in Saccharomyces cerevisiae
}

\author{
RUNE THOMSEN, ${ }^{1}$ DOMENICO LIBRI, ${ }^{2}$ JOCELYNE BOULAY, $^{2} \mathrm{MICHAEL} \mathrm{ROSBASH,}^{3}$ and \\ TORBEN HEICK JENSEN ${ }^{1}$ \\ ${ }^{1}$ Department of Molecular Biology, Aarhus University, 8000 Aarhus C., Denmark \\ ${ }^{2}$ Centre National de la Recherche Scientifique, Center de Genetique Moleculaire, 91190 Gif sur Yvette, France \\ ${ }^{3}$ Howard Hughes Medical Institute, Department of Biology, Brandeis University, Waltham, Massachusetts 02454, USA
}

\begin{abstract}
In the yeast Saccharomyces cerevisiae, a common conditional phenotype associated with deletion or mutation of genes encoding mRNA export factors is the rapid accumulation of mRNAs in intranuclear foci, suggested to be near transcription sites. The nuclear RNA exosome has been implicated in retaining RNAs in these foci; on deletion of the exosome component Rrp6p, the RNA is released. To determine the exact nuclear location of retained as well as released mRNAs, we have used mRNA export mutant strains to analyze the spatial relationship between newly synthesized heat shock mRNA, the chromosomal site of transcription, and known $S$. cerevisiae nuclear structures such as the nucleolus and the nucleolar body. Our results show that retained SSA4 RNA localizes to an area in close proximity to the SSA4 locus. On deletion of Rrp6p and release from the genomic locus, heat shock mRNAs produced in the rat7-1 strain colocalize predominantly with nucleolar antigens. Bulk poly(A) ${ }^{+} \mathrm{RNA}$, on the other hand, is localized primarily to the nuclear rim. Interestingly, the RNA binding nucleocytoplasmic shuttle protein Npl3p shows strong colocalization with bulk poly $(A)^{+}$RNA, regardless of its nuclear location. Taken together, our data show that retention occurs close to the gene and indicate distinct nuclear fates of different mRNAs.
\end{abstract}

Keywords: Nuclear mRNA retention; transcription-site foci; mRNA release; nuclear bodies

\section{INTRODUCTION}

The eukaryotic nucleus is structurally and functionally organized into nonmembranous subcompartments. The beststudied nuclear organelle is arguably the nucleolus, which is the major site of ribosome synthesis. Recently, the molecular composition and assembly of several other nuclear organelles have received attention in higher eukaryotes, and the function of such structures is starting to be revealed (Matera 1999). In the lower eukaryote Saccharomyces cerevisiae, the nucleolus has for a long time been the only described nuclear body. However, recently it was reported that $S$. cerevisiae cells have a nuclear body, through which Box C/D small nucleolar RNAs were shown to traffic (Samarsky et al. 1998; Verheggen et al. 2001). This body was named the nucleolar body and might resemble Cajal bodies from more complex cells. This is because a marker for these

Reprint requests to: Torben Heick Jensen, Department of Molecular Biology, Aarhus University, C.F. Møllers Alle, Bldg. 130, 8000 Aarhus C., Denmark; e-mail: thj@mbio.aau.dk.

Article and publication are at http://www.rnajournal.org/cgi/doi/ 10.1261/rna.5170303. structures, the human survival of motor neuron protein (SMN), localized to this nuclear entity on expression in yeast (Verheggen et al. 2001).

Nuclear mRNA export is a process facilitated by multiple factors, which exercise their roles at different steps in the pathway. In S. cerevisiae, several "mRNA export genes" have been identified, which can be roughly placed into three different categories: (1) "early" factors physically associated with the transcription process; (2) "intermediate" factors that accompany the mRNP through the nucleoplasm to the nuclear pore complex (NPC); and (3) "late", primarily static, factors that associate with the NPC. Obviously, these categories might show some overlap, for example, one factor might belong to two or more groups.

Conditional mutation or deletion of prominent mRNA export factors result in nuclear accumulation of poly $(\mathrm{A})^{+}$ RNA within a few minutes after shifting to the restrictive condition. Based on such criteria, early factors include members of the THO complex, which is functionally linked to the transcription machinery (Chavez and Aguilera 1997; Chang et al. 1999; Libri et al. 2002; Strasser et al. 2002; Zenklusen et al. 2002). The biochemical function of this complex is uncertain, but it might serve as a platform onto 
which the mRNA export factors Sub2p and Yra1p can bind (Libri et al. 2002; Strasser et al. 2002; Zenklusen et al. 2002). These two proteins have been shown by chromatin immunoprecipitation (ChIP) assays to associate with mRNA while it is still in close proximity to chromatin (Lei et al. 2001; Zenklusen et al. 2002). On the basis of similar experimental evidence, the mRNA binding nucleocytoplasmic shuttle protein $\mathrm{Npl} 3 \mathrm{p}$ might also be an early factor (Lei et al. 2001). Yra1p is believed to recruit the Mex67p/Mtr2p heterodimer, probable intermediate factors, which in turn mediates contact of the mRNP to late factors (Reed and Hurt 2002). These are largely NPC components, of which two well-studied members are the nucleoporins Nup159p/Rat7p and Nup42p/Rip1p.

We recently discovered that a block to nuclear export leads to rapid effects on the subnuclear localization of two heat shock mRNAs (hs-mRNAs), SSA4 and HSP104 (Jensen et al. 2001b). In a number of temperature-sensitive (ts) export mutants derived from all three groups, each mRNA was sequestered in a single intranuclear focus at the nonpermissive temperature (Hilleren et al. 2001; Jensen et al. 2001a,b; Libri et al. 2002). The relationship of a focus to different subnuclear structures and to its gene of origin has not been analyzed in detail. In all known cases, however, this retention requires Rrp6p as well as other components of the nuclear exosome, a large complex of exonucleolytic enzymes; for example, deletion of the nonessential RRP6 gene leads to release of hs-mRNA from intranuclear foci.

In this paper, we investigate further the nuclear locale of retained as well as released hs-mRNAs. Consistent with previous interpretations, we show here that the nuclear-retained SSA4 RNA signal is indeed localized at or adjacent to its corresponding gene locus. Deletion of RRP6 causes the majority of SSA4 RNA to colocalize with nucleolar antigens, whereas bulk poly $(\mathrm{A})^{+}$RNA is localized at the nuclear rim.

\section{RESULTS}

\section{Export-inhibited mRNAs accumulate in a nuclear locale overlapping DNA}

To determine the nuclear location of retained mRNAs, we incubated yeast mRNA export mutants $\Delta$ rip 1 or rat7-1 at their restrictive temperatures for $30 \mathrm{~min}$ and subjected fixed cells to triple-color-labeling studies: fluorescent in situ hybridization (FISH) for RNA, immunolocalization of the nucleolar body or the nucleolus, and DAPI staining for DNA. RNAs were detected either with Cy3-labeled probes specific for SSA4 RNA or with a Cy3-labeled $\mathrm{dT}_{70}$ probe for poly $(\mathrm{A})^{+}$RNA. To visualize the nucleolar body, we introduced a plasmid expressing the human SMN protein fused to GFP into $\Delta$ rip1 cells. For detection of the nucleolus, cells were stained with antibodies directed toward the nucleolar protein Mpp10p.

As previously observed (Jensen et al. 2001b), SSA4 RNA appeared as a single nuclear dot on transcriptional induction in the $\Delta$ rip1 haploid strain (Fig. 1A,C). In contrast, bulk mRNA, as visualized by the dT probe, stained a larger fraction of the nucleus in both $\Delta$ rip 1 and rat7-1 strains (Fig. $1 \mathrm{~B}, \mathrm{D})$. Consistent with the report from Verheggen et al. (2001), SMN-GFP accumulated to a restricted area (the nucleolar body) within nuclei of individual cells (Fig. 1A,B). Neither SSA4 nor poly (A) ${ }^{+}$RNA localized to the SMN-GFP stained area but, rather, showed physical overlap with the DAPI stain (Fig. 1A,B).

A comparable result was obtained when mRNA subnuclear localization was compared with the nucleolus. In the $\Delta$ rip1 strain, SSA4 RNA overlapped with the DAPI stain but not the nucleolus, as defined by Mpp10p (Fig. 1C). An identical result was obtained when we assayed for HSP104 RNA localization in the $\Delta$ ripl strain (data not shown). Furthermore, both hs-mRNAs showed a similar localization pattern (nonoverlapping with Mpp10p) in the rat7-1 mRNA export mutant (data not shown). Poly (A) ${ }^{+}$RNA in the rat7-1 strain mainly localized to an area covered by DAPI stain, although a modest overlap with the nucleolus was detectable (Fig. 1D). A similar result was obtained when the $\Delta$ ripl strain was used (data not shown). We conclude that mRNA retained within the nucleus of the $\Delta$ ripl and rat7-1 strains is largely separate from the nucleolus and the nucleolar body.

\section{The SSA4 RNA nuclear accumulation site coincides with the SSA4 locus}

Having established colocalization with DNA by DAPI staining, we wanted to evaluate the nuclear position of retained SSA4 RNA at a higher resolution. For this purpose, we created two different $\Delta$ ripl strains that both express tetRGFP fusion proteins. They bind and label tetO operator sites, inserted into the genome in close proximity to the SSA4 locus on chromosome V. The $\Delta$ rip1/URA3-tetO/tetRGFP strain harbors 336 tetO operator sites inserted into the URA3 locus on the left arm of chromosome $\mathrm{V}, \sim 250 \mathrm{~kb}$ upstream of SSA4. The $\Delta$ rip1/BMH1-tetO/tetR-GFP strain contains 448 tetO operator sites at the BMH1 locus on the right arm of chromosome $\mathrm{V}, \sim 180 \mathrm{~kb}$ downstream of SSA4 (Fig. 2A).

Hs-mRNA expression was induced in both strains, and cells were fixed and assayed for localization of SSA4 RNA and tetR-GFP by dual SSA4 RNA-FISH/GFP-immunofluorescence analysis. Representative cells from the experiment are shown in Figure 2B (upper and middle rows), which illustrates that the localization of SSA4 RNA in these strains is in very close proximity to both the $B M H 1$ and URA3 loci. As a control, RNA-FISH for HSP104 RNA was also performed in the $\Delta$ rip1/URA3-tetO/tetR-GFP strain. AsHSP104 is on chromosome XII, overlap between retained HSP104 RNA and the URA3 gene was neither expected nor found (Fig. 2B, lower row). Moreover, we consistently ob- 
A

$\Delta$ rip1 $42^{\circ} \mathrm{C}, 30^{\prime}$
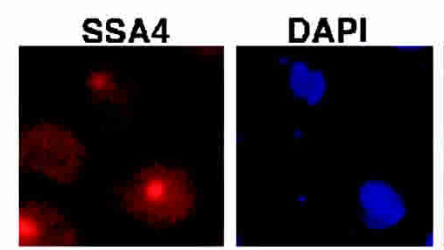

SSA4/DAPI
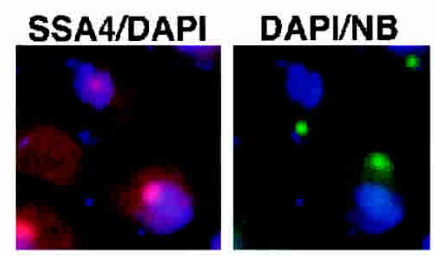

C

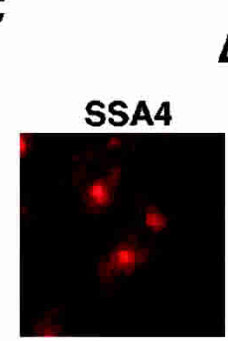

$\Delta$ rip1 $42^{\circ} \mathrm{C}, 30^{\prime}$

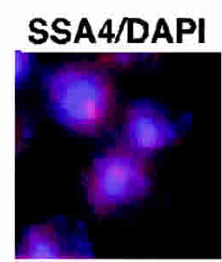

DAPI

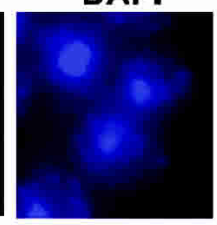

DAPINUC

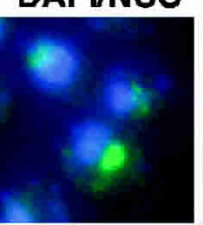

NB

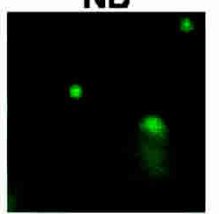

SSA4/NB

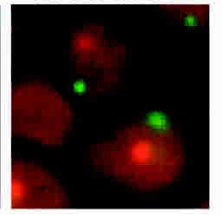

B

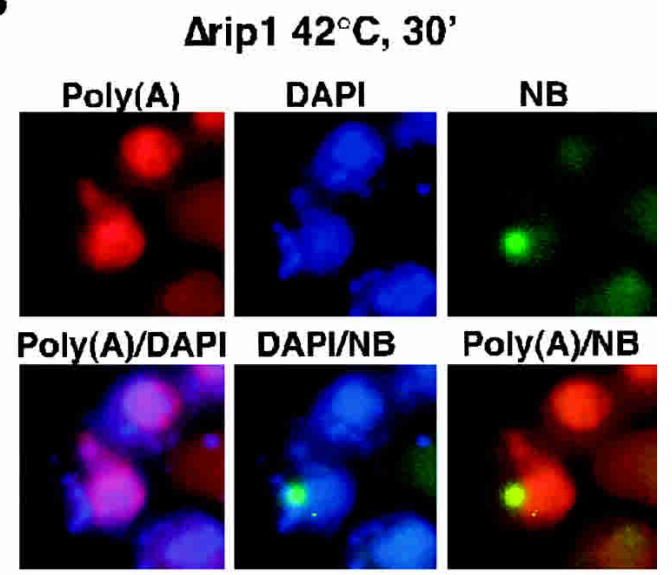

D

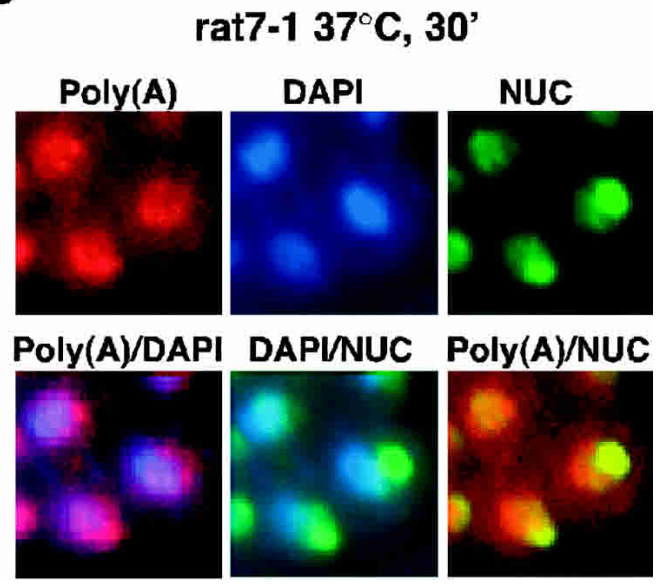

FIGURE 1. Localization of retained $S S A 4$ and poly $(A)^{+}$RNA within nuclei of mRNA export mutant cells. $(A, B)$ Triple-color RNA-FISH, SMN-GFP immunolocalization, and DAPI staining analysis on fixed $\Delta$ rip1 cells after a 30 -min incubation at $42^{\circ} \mathrm{C}$. SSA4 $(A)$ and poly $(\mathrm{A})^{+} \mathrm{RNA}$ (B) was visualized with Cy3-labeled KD199/KD200 probes and a Cy3-labeled oligo-dT 70 probe, respectively. Cells were transformed with a plasmid expressing human SMN-GFP protein, which was visualized with an anti-GFP antibody. DNA was stained with DAPI. (C) Triple-color SSA4 RNA-FISH, Mpp10p immunolocalization, and DAPI staining analysis of the $\Delta$ rip1 strain treated as in $A$ and B. Mpp10p was detected using an Mpp10p-specific antibody. (D) Triple-color poly(A) ${ }^{+}$RNA-FISH, Mpp10p immunolocalization, and DAPI staining analysis on fixed rat7-1 cells after a $30-$ min incubation at $37^{\circ} \mathrm{C}$. Individual images were merged as indicated. (NB) Nucleolar body; (NUC) nucleolus.

served two distinct nuclear dots per cell with dual SSA4/ HSP104 RNA-FISH analysis, using Cy3-labeled probes directed against both SSA4 and HSP104 RNA (Fig. 2D).

To determine the statistical significance of our observations, the distance between the signals for SSA4 or HSP104 RNA and the BMH1 or URA3 loci were determined for a substantial number of cells from each experiment (the distributions are plotted in Fig. 2C to the right side of the relevant images). Differences between distance distributions for the individual strains were statistically significant $(P<0.001)$, indicating that even a difference of $70 \mathrm{~kb}$ (the difference between SSA4-URA3 and SSA4-BMH1 physical distances) can be reliably detected.

The $\Delta$ rip 1 mutant is classified as group iii, or late. Because the majority if not all of Riplp is localized to the cytoplasmic site of the nuclear membrane, it is unlikely to shuttle and is therefore probably a static, nuclear pore- associated protein (Strahm et al. 1999; Rout et al. 2000). We wanted to determine if SSA4 RNA produced in an "early" export mutant would also colocalize to its own site of synthesis. For this purpose, a $\Delta h p r 1 / \mathrm{BMH} 1$-tetO/tetR-GFP strain was constructed. We have previously shown that hsmRNA is retained in the nucleus of a $\Delta h p r 1$ strain at $37^{\circ} \mathrm{C}$ (Libri et al. 2002). Hprlp is a member of the THO complex, which is thought to act cotranscriptionally and therefore qualifies as an "early" export factor (Strasser et al. 2002; Zenklusen et al. 2002). Newly synthesized SSA4 RNA was linked to the SSA4 locus in the $\Delta h p r 1 / \mathrm{BMH} 1$-tetO/tetRGFP strain, with a distribution of measured distances very similar to that of SSA4 RNA in the $\Delta$ rip 1 background (Fig. 2E,F; compare distribution to that of Fig. 2C). We conclude that nuclear-retained SSA4 RNA accumulates in close proximity to its site of transcription, independent of whether an "early" or a "late" mRNA export mutant is used. 
A

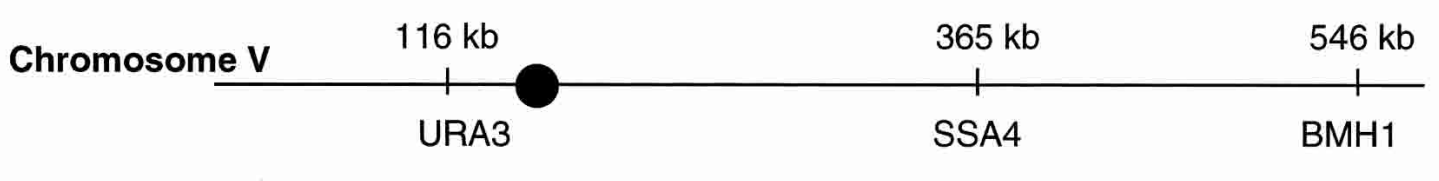

B
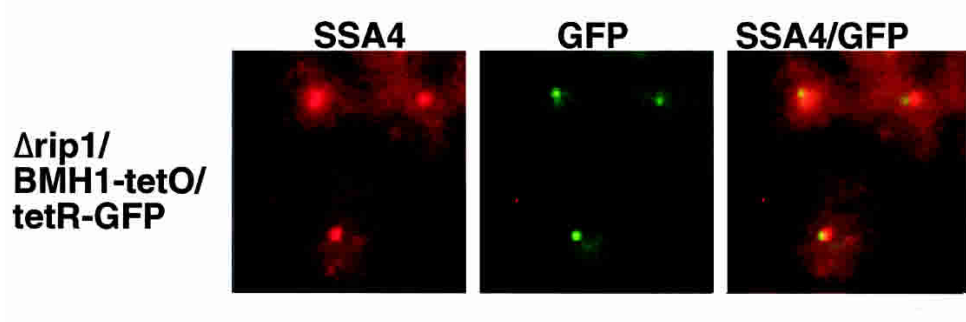

C
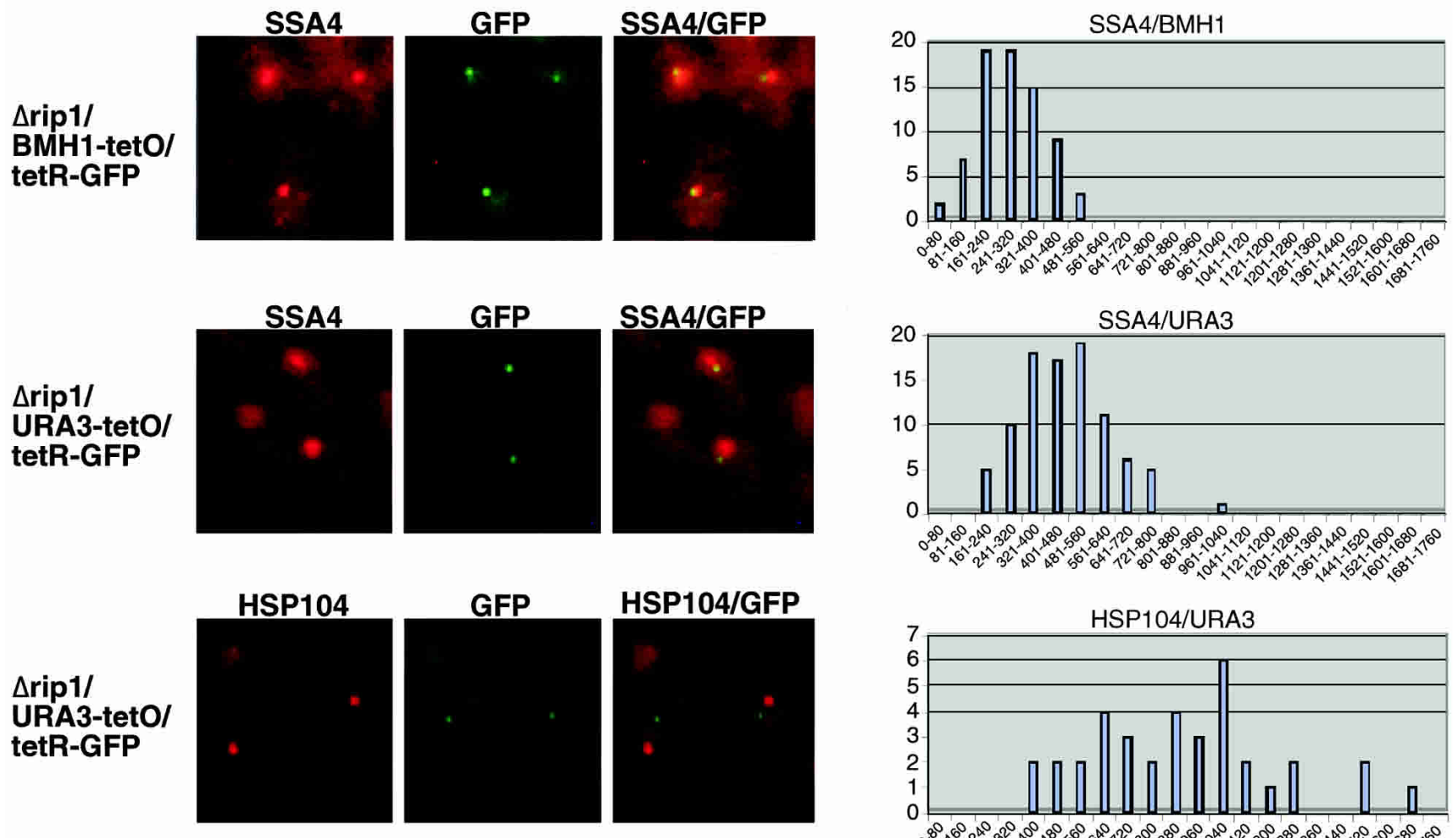

D
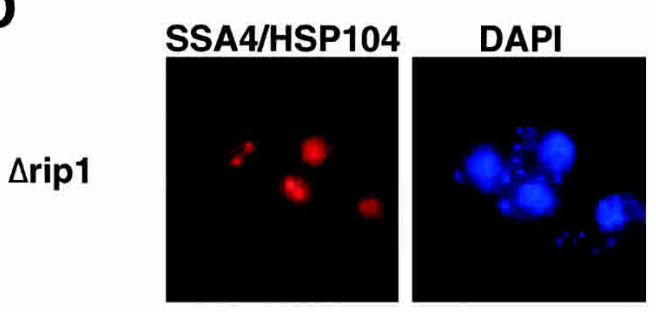

E
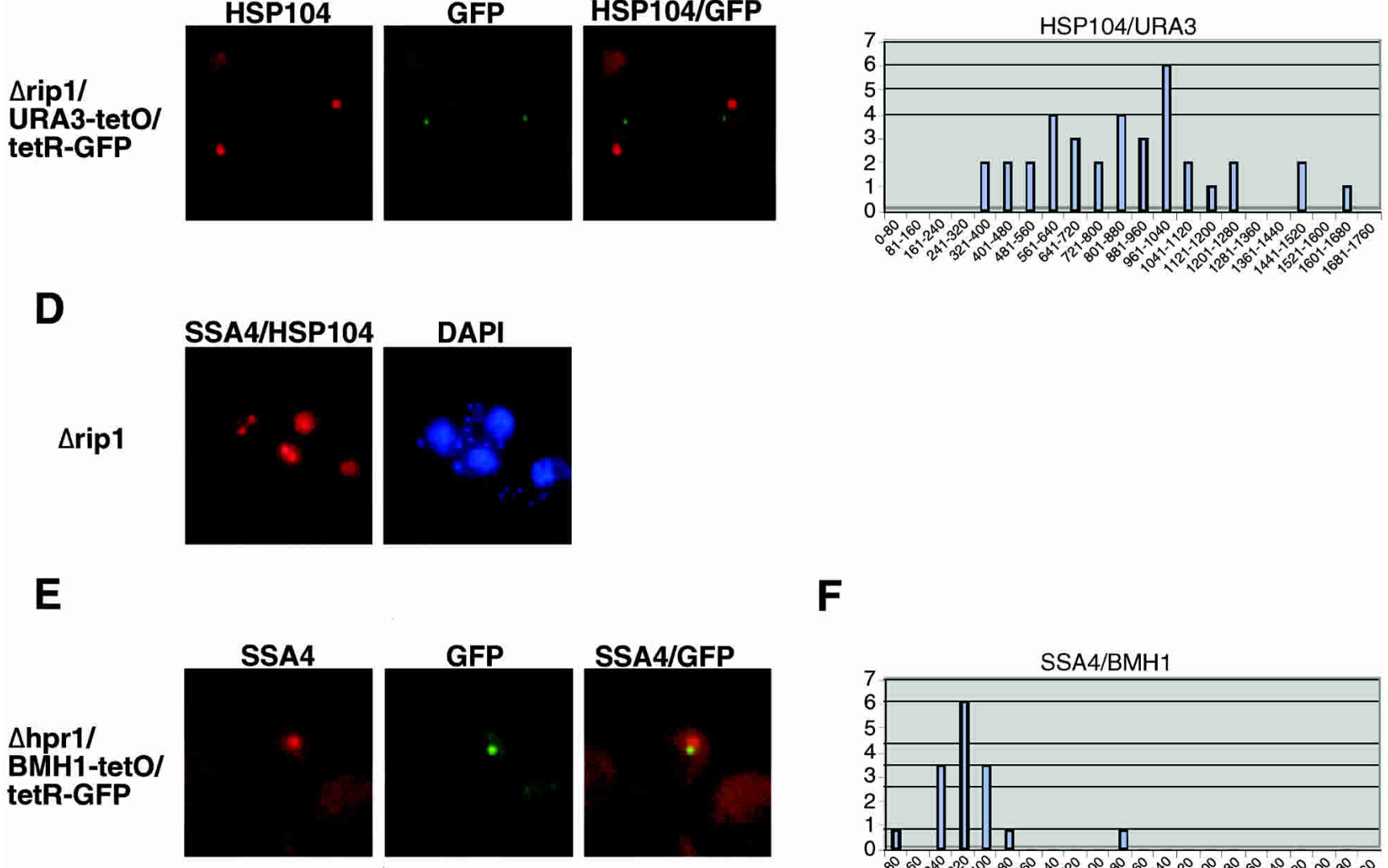

F

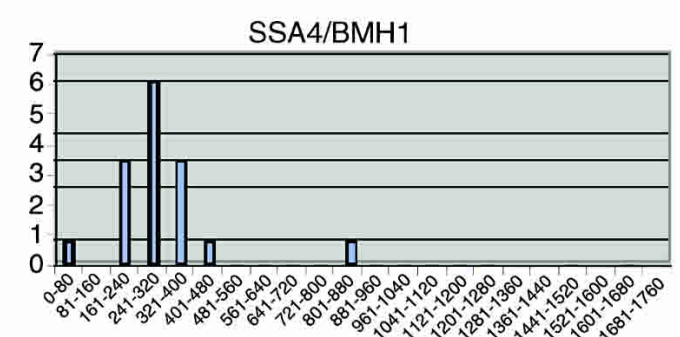

FIGURE 2. (Legend on facing page) 


\section{Nuclear mRNA localization on deletion of RRPG}

We have previously shown that transcript retention in nuclear foci requires components of the nuclear exosome (Hilleren et al. 2001; Jensen et al. 2001a; Libri et al. 2002). For example, addition of an RRP6 deletion (Rrp6p is strictly nuclear and defines the nuclear exosome; Butler 2002) to a late mRNA export mutant (e.g., $\Delta$ rip1 or rat7-1) eliminates transcript retention within foci. In such a double-mutant background, mRNA is not retained and presumably proceeds toward the cytoplasm (Hilleren et al. 2001; data not shown). However, an export block at the nuclear envelope persists at the restrictive temperature (Figs. 3, 4; Hilleren et al. 2001).

To assay the localization of a released hs-mRNA, we performed double staining for HSP104 RNA and Mpp10p in

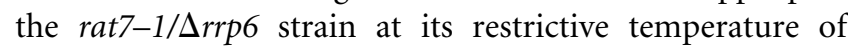
$37^{\circ} \mathrm{C}$. Under these conditions, HSP104 RNA localized to an area of the nucleus largely devoid of DAPI stain but considerably overlapping with Mpp10p staining (Fig. 3, upper panels). Because some dispersion of the Mpp10p signal was evident in this strain background as compared with a wildtype strain (data not shown), we also performed double staining for HSP104 and another nucleolar component, Noplp. This protein showed a more restricted localization than Mpp10p, indicating that different components of the nucleolus might localize somewhat differently under these conditions (Fig. 3, lower panels). Regardless, the Nop1p signal is contained within the HSP104-stained area of the DNA-excluded part of the nucleus. Thus, the bulk of released HSP104 RNA colocalizes with nucleolar antigens when restricted from entering the cytoplasm. Comparable results were obtained when using a $\Delta$ rip $1 / \Delta r r p 6$ strain at its restrictive temperature of $42^{\circ} \mathrm{C}$, and when assaying for $S S A 4$ RNA instead of HSP104 RNA (data not shown).

In contrast to these two hs-mRNAs, total poly $(\mathrm{A})^{+} \mathrm{RNA}$ localizes to the nuclear periphery in rat7-1/Drrp6 cells 30 min after the shift to the restrictive temperature, as previously shown (Hilleren et al. 2001). In a rat7-1 single mutant, the poly $(\mathrm{A})^{+}$signal shows a strong intranuclear granular staining. These observations have been interpreted to reflect a large number of transcription sites; release coupled with a persistent export block at the nuclear periphery leads to staining of the nuclear rim in the double-mutant strain. We imagined that this phenomenon might allow for the identification of proteins, which associate with the mRNP cotranscriptionally and stay attached to the released mRNP until arrival at the NPC. Such factors should colocalize with poly $(\mathrm{A})^{+}$RNA. To test this hypothesis, we used antibodies against the nucleocytoplasmic shuttling protein Npl3p, which has tested positive in ChIP assays and has been shown to be a major mRNA binding protein in yeast (Flach et al. 1994; Lei et al. 2001). The rat7-1 and rat7-1/4rrp6 strains were grown at the permissive temperature of $25^{\circ} \mathrm{C}$ and shifted to the restrictive temperature of $37^{\circ} \mathrm{C}$. Cells were then fixed at different times following the temperature shift and subjected to double-poly(A) ${ }^{+}$RNA-FISH and Npl3p immunostaining.

At all times and in both strains, poly $(\mathrm{A})^{+} \mathrm{RNA}$ and Npl3p showed considerable overlap (Fig. 4). This observation indicates that the majority of Npl3p in the cell is associated with mRNA. In rat7-1 cells, the poly(A) ${ }^{+} \mathrm{RNA} / \mathrm{Npl} 3 \mathrm{p}$ signal remained in intranuclear granules throughout the course of the experiment (Fig. 4, left panels). This pattern

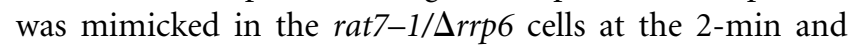
5-min time points (Fig. 4, right panels, two upper rows). However, at later times after the temperature shift (i.e., 10 and $30 \mathrm{~min}$ ), the majority of poly $(\mathrm{A})^{+}$RNA and Npl3p was associated with the nuclear periphery (Fig. 4, right panels, two lower rows). Because poly(A) ${ }^{+}$RNA and Npl3p move from intranuclear foci to the nuclear periphery with comparable kinetics, we suggest that the observations reflect cotranscriptional recruitment of Npl3p to nascent mRNA followed by migration of the mRNP to the closed nuclear envelope.

\section{DISCUSSION}

In this paper, we have used ts mutants and FISH to analyze mRNA localization and mRNP trafficking within the yeast nucleus. We had previously localized newly synthesized hsmRNA to an intranuclear dot in several yeast mRNA export mutants (Jensen et al. 2001b). A diploid strain, deleted for

FIGURE 2. Colocalization of retained SSA4 RNA with nearby gene loci in mRNA export mutants $\Delta$ ripl and $\Delta h p r 1$. (A) Schematic representation of a portion of yeast chromosome $\mathrm{V}$ showing the distances of the URA3, SSA4, and BMH1 loci relative to the beginning of the left arm. Distances are given in kilobases $(\mathrm{kb})$ and the position of the centromere is denoted by a black dot. (B) Dual-color SSA4 or HSP104 RNA-FISH combined with tetR-GFP immunolocalization analysis on fixed $\Delta$ rip1/BMH1-tetO/tetR-GFP or $\Delta$ rip1/URA3-tetO/tetR-GFP cells after a 30-min incubation at $42^{\circ} \mathrm{C}$. Relevant strain and hs-mRNA target is indicated to the left and above the images, respectively. SSA4 and HSP104 RNAs were visualized using KD199/KD200 and THJ203/THJ204/THJ205/THJ206 probes, respectively. TetR-GFP was detected by an anti-GFP antibody. (C) Distribution of the number of RIP1 deleted cells as a function of the distances between SSA4 or HSP104 RNA foci and BMH1 or URA3 loci as indicated above the graphs. Analyzed cells were divided into groups on the basis of the distance between the respective RNA and DNA dots. Distance intervals in nanometers $(\mathrm{nm})$ are given on the horizontal axes. Numbers of analyzed cells belonging to the appropriate group are given on the vertical axes. To make an approximate conversion of measured distance units into nanometers, we set the average size of a yeast cell to $5 \mu \mathrm{m}$. $(D)$ Double SSA4 and HSP104 RNA-FISH on fixed $\Delta$ rip1 cells after a 30 -min incubation at $42^{\circ} \mathrm{C}$. RNAs were visualized as described in $B$. DNA was stained with DAPI. (E) Dual-color SSA4 RNA-FISH and tetR-GFP immunolocalization analysis on fixed $\Delta h p r 1 / \mathrm{BMH} 1$-tetO/tetR-GFP cells after a 20-min incubation at $37^{\circ} \mathrm{C}$. SSA4 RNA and tetR-GFP were visualized as described in $B$. (F) Distribution of the number of HPR1 deleted cells as a function of the distance between the SSA4 RNA focus and the BMH1 locus. Cells were analyzed and depicted as described in C. 
rat7-1

$42^{\circ} \mathrm{C} 60^{\prime}$
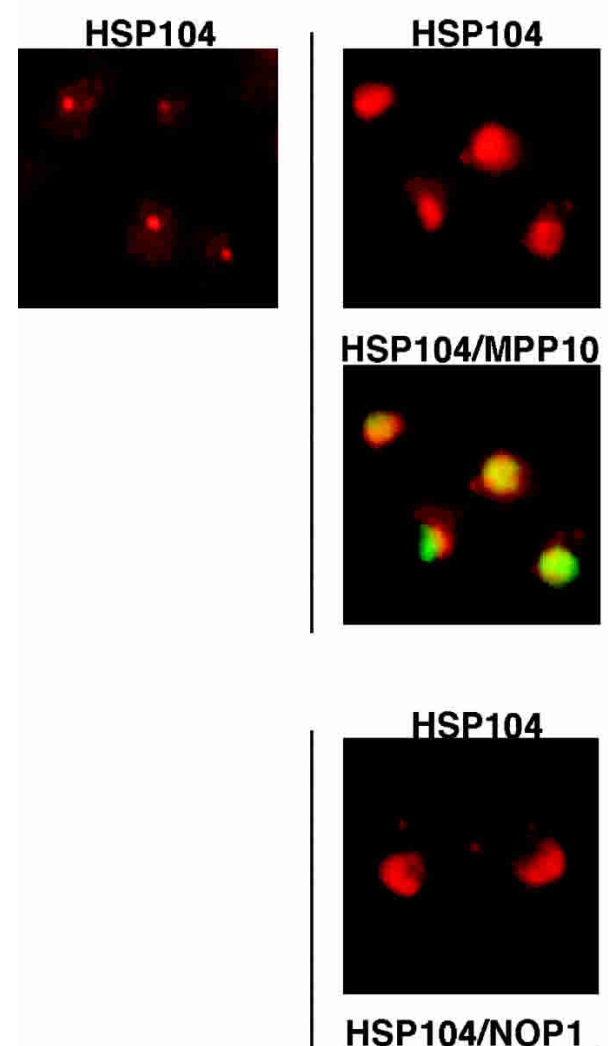

rat7-1/4rrp6 $42^{\circ} \mathrm{C} 60^{\prime}$
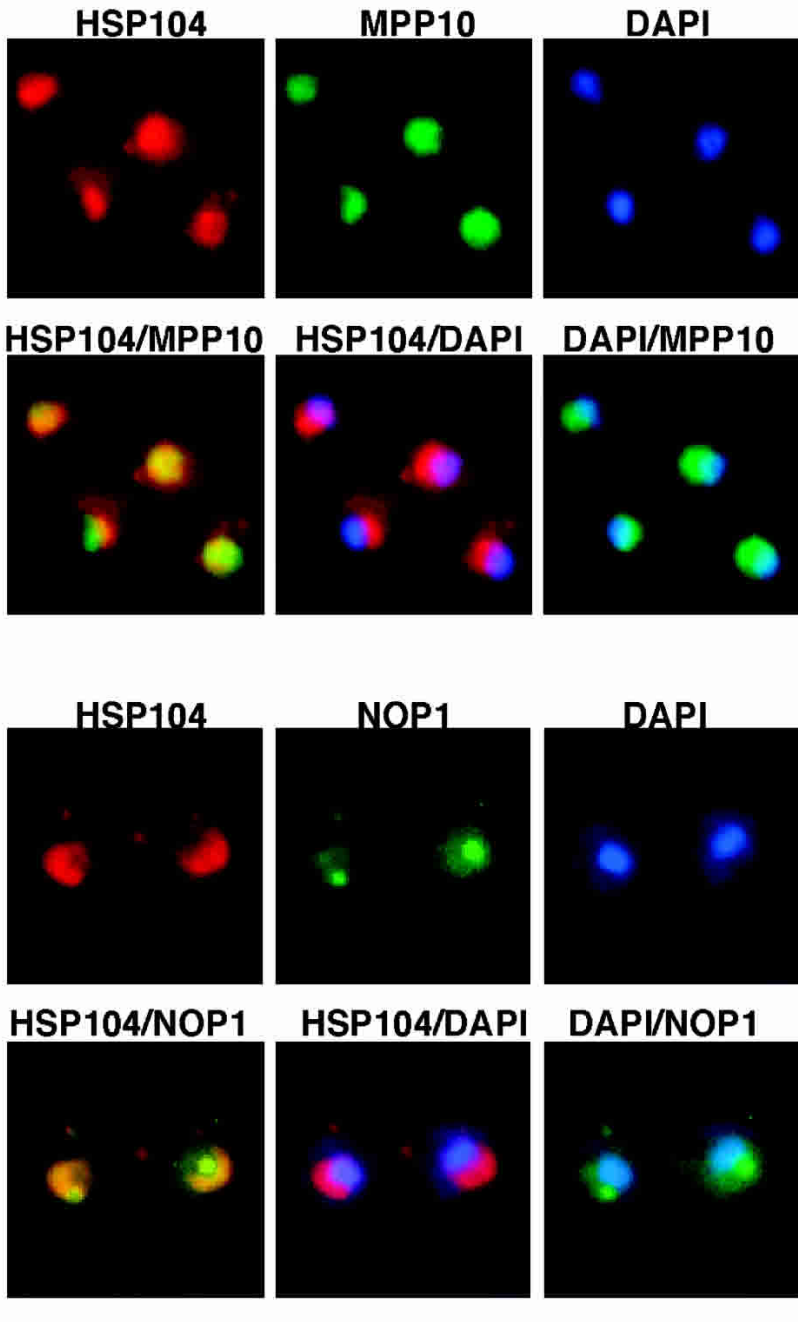

DAP

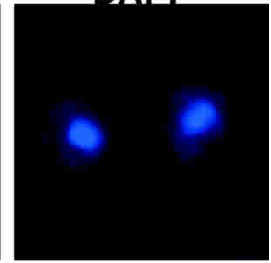

DAPINOP1

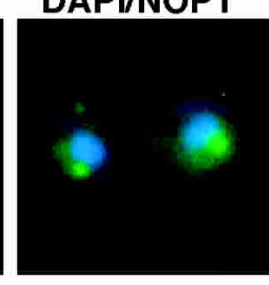

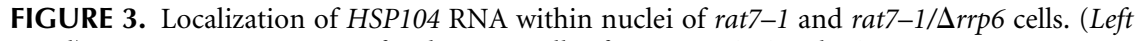
panel) HSP104 RNA-FISH on fixed rat7-1 cells after a 60 -min incubation at $42^{\circ} \mathrm{C}$. Image was constructed as a comparison to right panels. (Right panels) Triple-color HSP104 RNA-FISH, Mpp10p (upper panels) or Nop1p (lower panels) immunolocalization and DAPI staining analysis in the rat7-1/Drp6 strain after a 60 -min incubation at $42^{\circ} \mathrm{C}$. HSP104 RNA, Mpp10p, and DNA were visualized as described in the legend to Figure 2. Noplp was detected using an Noplp-specific antibody.

both copies of the RIP1 gene, contained two dots. These observations indicated that localization of the retained RNA was in close proximity to its site of transcription, which is strongly supported by data presented in this paper.

We have tested a late $(\Delta$ rip 1$)$ export mutant and find significant colocalization of retained SSA4 RNA with tetRGFP molecules tethered to the nearby BMH1 and URA3 loci present on the same chromosome. The average distance measured between the SSA4 RNA signal and the tagged loci was significantly smaller than the average distance measured between the BMHI locus and nuclear-retained HSP104 RNA, expressed from an unlinked locus on chromosome XII. Furthermore, we were able to detect a statis-

tically significant difference in the distances measured between SSA4 RNA and $B M H 1$ compared with the distances measured between SSA4 RNA and $U R A 3$, all of which are on chromosome $\mathrm{V}$. The latter distance is roughly 1.7 times the former, which correlates well with the differences in physical distances (1.4 times). Finally, we have crossed the two GFP-tagged strains and measured the distance between the URA3 and $B M H 1$ loci; the average distance between the two GPP spots is exactly the sum of the SSA4/URA3 and SSA4/ $B M H 1$ average distances (data not shown). We therefore conclude that the dot within which SSA4 RNA accumulates on transcriptional induction in the $\Delta$ rip1 strain is at or very near its transcription site. The distribution of distances between SSA4 RNA and the $B M H 1$ locus in the $\Delta h p r 1$ strain mirrors the one observed in the $\Delta$ rip1 strain, strongly indicating that a similar RNA dot also accumulates in "early" export mutants. Although we expect the hsmRNA-containing dots observed in other mRNA export mutants will be similar, the lack of a high resolution view makes it impossible to tell whether all mutants will give rise to identical dots or whether there are subtle differences between the dot induced in $\Delta$ rip 1 and the dot induced in $\Delta h p r 1$. It would be of interest in this context to integrate a second SSA4 gene at different distances from the SSA4 locus, to try to address whether two closely spaced genes make one or two dots.

Although the generality of the dot formation phenomenon still needs to be addressed, we have so far observed it consistently for HSP104, SSA4, and GAL1 RNAs; we have not tried other probes (Jensen et al. 2001b). As all three of these genes are highly inducible, it is possible that robust transcription is required to saturate locally the release system and observe a phenotype. This issue is also relevant to the granular appearance of the poly $(\mathrm{A})^{+}$signal under dot conditions, that is, how many transcripts and dots does this reflect: just abundantly transcribed mRNAs or all transcripts?

Consistent with the retention of SSA4 RNA close to chromatin, we observe no colocalization of RNA with two other yeast nuclear bodies: the nucleolus and the nucleolar body. It has been reported that conditions of high temperature for 


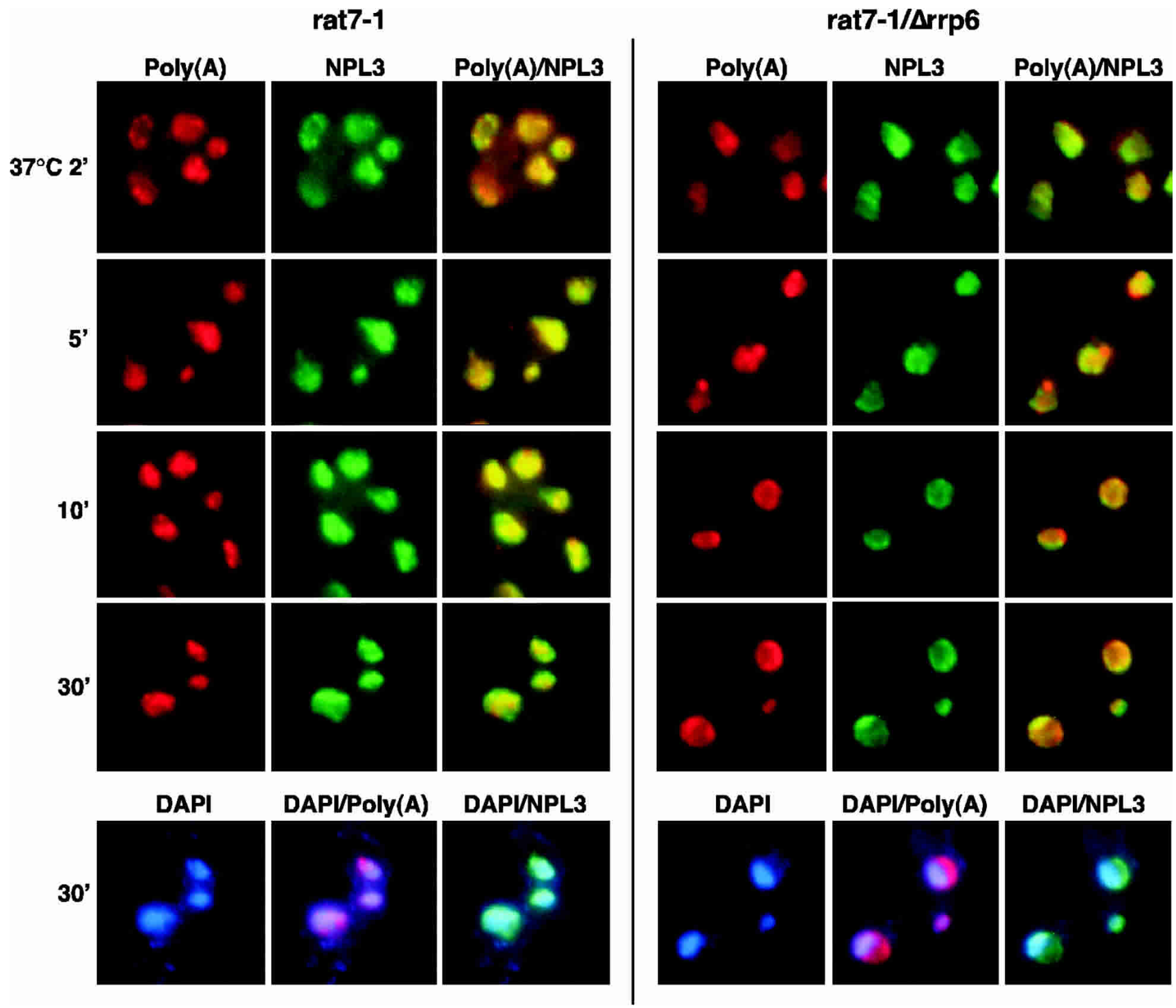

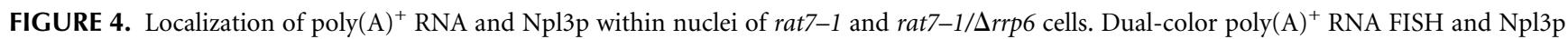

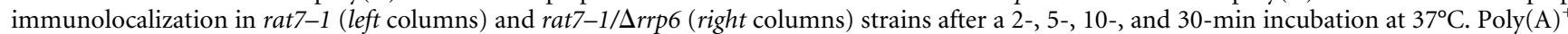
RNA and Npl3p were visualized using a Cy3-labeled oligo $(\mathrm{dT})_{70}$ and an Npl3p-specific antibody, respectively. DAPI stain of both sets of cells from the 30 -min time point was merged to the poly $(\mathrm{A})^{+}$and Npl3p signals (lower rows).

prolonged time $(2-3 \mathrm{~h})$ disassembles the nucleolus, causing several nucleolar proteins to relocate to the cytoplasm (Liu et al. 1996). Indeed, we also observe some dispersion of nucleolar antigens (Mpp10p and Nop1p) on a temperature shift, whereas the morphology of the SMN-GFP signal is undisturbed (data not shown). However, by restricting the incubation time at $42^{\circ} \mathrm{C}$ to $1 \mathrm{~h}$ or less, we were able to obtain cells with distinct hs-mRNA signals and well-defined nucleoli.

On deletion of RRP6 in the rat7-1 export mutant background, a majority of nuclear-restricted SSA4 and HSP104 hs-mRNA now accumulate in a subregion of the nucleus, which overlaps with the nucleolar markers Mpp10p and Nop1p. The localization is not absolute, consistent with the fact that some Hsp104p protein synthesis continues in the

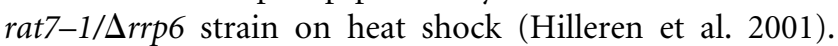
Nonetheless, there is a clear difference in this strain between the two hs-mRNAs localizations and bulk poly $(\mathrm{A})^{+}$RNA. The latter is primarily detected at the nuclear envelope, as previously described (Hilleren et al. 2001). Perhaps the difference indicates that hs-mRNA normally follows a nucleolar exit route. Another possibility is that the high abundance of the hs-mRNAs gives rise to an aberrant nucleolar accumulation in the mutant strain background. The transcriptional response to heat shock is extremely robust; that is, large amounts of hs-mRNA are rapidly synthesized. Perhaps as a consequence, most if not all of this RNA is aberrant in the export mutant strains, evident by the long poly(A)-tails 
in the rat7-1 and $\Delta$ rip1 backgrounds (Jensen et al. 2001b). Such aberrant and abundant hs-mRNA might overwhelm the nucleoplasmic degradation as well as transport systems and lead in some enigmatic way to nucleolar accumulation. In this view, less abundant (and perhaps less aberrant) mRNA species might continue on their normal path toward the nuclear envelope.

Npl3p, which is a putative structural component of functional mRNPs (Lee et al. 1996) associates with bulk poly $(\mathrm{A})^{+} \mathrm{RNA}$, and Npl3p antibodies are capable of precipitating chromatin in ChIP experiments (Lei et al. 2001).

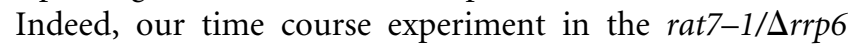
strain indicates that Npl3p is recruited to these RNAs cotranscriptionally and stays associated at least until their arrival at the nuclear rim (Fig. 4). This observation appears at odds with recent in vivo cross-linking studies, which indicated that Npl3p no longer associates with non-hs poly(A) ${ }^{+}$ RNA under heat shock conditions (Krebber et al. 1999). One possibility is that the RNA at the nuclear rim is largely hs-mRNA, that is, only a subset of hs-mRNA (including SSA4 and HSP104) heads to the nucleolus in the doublemutant strains. This begs the question, what fraction of the nuclear poly $(\mathrm{A})^{+}$signal reflects hs-mRNA under heat shock conditions? We favor the simplest answer, namely, a small fraction. This means that the nucleolus-nuclear rim distinction largely divides the nuclear RNA population into heat shock and non-heat shock subsets. To reconcile this interpretation with the Npl3p cross-linking results, we suggest that the changes in Npl3p interactions with non-hs-mRNAs on heat shock reflect relatively subtle changes in cross-linking efficiency rather than association per se.

A possible distinction between hs- and non-hs-mRNA at the level of mRNA export is controversial, and we have favored the notion that there is little difference between the export pathway taken by hs- and non-hs-mRNAs (Vainberg et al. 2000). The different localization results shown here for two hs-mRNAs on the one hand and poly $(\mathrm{A})^{+}$RNA on the other indicate that there is indeed some differences in
mRNP composition or arrangement between mRNP populations that might impact nuclear trafficking events. Whether these differences only reflect the mutant circumstances or are also true in wild-type cells is a matter for further investigations.

\section{MATERIALS AND METHODS}

\section{Yeast strains}

Yeast strains used in this study are shown in Table 1. $\Delta$ rip1 strains expressing TetR-GFP and harbouring tetO operator sites inserted at the $B M H 1$ and $U R A 3$ loci, respectively, were generated by genetic crosses between $\Delta$ rip1 and the K6745 and K7022 strains (Ciosk et al. 1998). The $\Delta h p r 1 / B M H 1$-tetO/tetR-GFP strain was constructed by crossing $\Delta h p r 1$ to K7022.

\section{In situ hybridization and immunolocalization analysis}

Fixation and preparation of cells for FISH analysis were done as previously described (Jensen et al. 2001b). RNAs were detected using the following previously described Cy3-labeled oligonucletide probes: THJ203, THJ204, THJ205, and THJ206 for HSP104, KD199 and KD200 for SSA4, and a Cy3-labeled oligo(dT) ${ }_{70}$ for poly $(\mathrm{A})^{+}$RNA, respectively (Jensen et al. 2001b).

For combined RNA-FISH/immunolocalization analysis, cells were first processed for FISH as described (Jensen et al. 2001b). Instead of mounting immediately, cells were washed once for 5 $\min$ at room temperature in wash buffer (PBS, 0.1\% Triton $\mathrm{X}-100$ ). Subsequently, cells were incubated for $30-60 \mathrm{~min}$ at $37^{\circ} \mathrm{C}$ with relevant primary antibody diluted in wash buffer, followed by three washes in wash buffer for $5 \mathrm{~min}$ at room temperature. After the final wash, relevant secondary antibody diluted in wash buffer was added for $30-60 \mathrm{~min}$ at $37^{\circ} \mathrm{C}$, again followed by three washes in wash buffer for $5 \mathrm{~min}$ at room temperature. Finally, cells were mounted in mounting media as described (Jensen et al. 2001b).

\section{Imaging}

Images were acquired on an Olympus BX51 microscope equipped with a cooled Olympus DP50 CCD camera and analySIS software.

TABLE 1. Saccharomyces cerevisiae strains used in this study

\begin{tabular}{|c|c|c|}
\hline Strain & Genotype & Source \\
\hline yRP1579 & 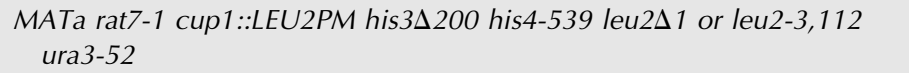 & Hilleren et al. 2001 \\
\hline yRP1585 & $\begin{array}{l}\text { MATa rat7-1 rrp6::URA3 cup1::LEU2PM ura3-52 leu2D1 or leu2-3,112 } \\
\text { trp1-1 his3 } 200 \text { and/or -539 }\end{array}$ & Hilleren et al. 2001 \\
\hline yRP1586 & MATa rip1::KAN ade2-1 ura3-1 his3-11,15 leu2-3,112 trp1-1 & Hilleren et al. 2001 \\
\hline yRP1587 & MATa rip1::KAN rrp6::URA3 leu2-3,112 trp1-1 ura3-1 or ura3-52 & Hilleren et al. 2001 \\
\hline W303s rip1 & MATa rip $1:: K A N$ ade2 his3 leu2 trp1 ura3 & Stutz et al. 1997 \\
\hline W303Arip1/URA3-tetO/terR-GFP & $\begin{array}{l}\text { Mato or Mata rip1::KAN ura3::URA3tetO112 leu2::LEU2 tetR-GFP trp1 } \\
\text { his3 }\end{array}$ & This study \\
\hline W303 $\Delta$ rip1/BMH1-tetO/tetR-GFP & $\begin{array}{l}\text { Mata or Mata rip1::KAN bmh1::URA2tetO224 leu2::LEU2 tetR-GFP trp1 } \\
\text { his3 }\end{array}$ & This study \\
\hline W303 $h$ pr1/BMH1-tetO/tetR-GFP & $\begin{array}{l}\text { Mato or Mata hpr1::KAN bmh1::URA2tetO224 leu2::LEU2 tetR-GFP trp1 } \\
\text { his3 }\end{array}$ & This study \\
\hline
\end{tabular}


Image handling was done in Adobe Photoshop. Distances between RNA and GFP-labeled DNA signals in the $\Delta$ ripl strain were measured for 74 cells (BMH1-SSA4 RNA), 92 cells (URA3 1-SSA4 RNA), and 36 cells (URA3-HSP104 RNA). In the hpr1 strain 18 cells (BMH1-SSA4 RNA) were analyzed. A student test was applied to assess the statistical significance of differences in the four sets of data. All distributions were statistically different $(P<0.001)$, with the exception of the BMH1-SSA4 RNA distance in $\Delta$ rip1 compared with the BMH1-SSA4 RNA distance in $\Delta h p r 1$.

\section{ACKNOWLEDGMENTS}

We thank the following for providing reagents: Rafal Ciosk and Kim Nasmyth (yeast strains K6745 and K7022), Francois Dragon and Susan Baserga (Mpp10p antibody), Pam Silver (GFP antibody), Reinhard Luhrmann (Npl3p antibody), John Aris (Noplp antibody), and Edouard Bertrand (SMN-GFP expression plasmid). We are grateful to Ken Dower for technical advice and fruitful discussions as well as to Melissa Moore and Christian K. Damgaard for comments on the manuscript. This work was supported by grants from the CNRS (D.L.), the NIH (M.R.), and the Danish Research Council (T.H.J.).

The publication costs of this article were defrayed in part by payment of page charges. This article must therefore be hereby marked "advertisement" in accordance with 18 USC section 1734 solely to indicate this fact.

Received January 31, 2003; accepted May 21, 2003.

\section{REFERENCES}

Butler, J.S. 2002. The yin and yang of the exosome. Trends Cell Biol. 12: 90-96.

Chang, M., French-Cornay, D., Fan, H.Y., Klein, H., Denis, C.L., and Jaehning, J.A. 1999. A complex containing RNA polymerase II, Paflp, Cdc73p, Hprlp, and Ccr4p plays a role in protein kinase C signaling. Mol. Cell. Biol. 19: 1056-1067.

Chavez, S. and Aguilera, A. 1997. The yeast HPR1 gene has a functional role in transcriptional elongation that uncovers a novel source of genome instability. Genes \& Dev. 11: 3459-3470.

Ciosk, R., Zachariae, W., Michaelis, C., Shevchenko, A., Mann, M., and Nasmyth, K. 1998. An ESP1/PDS1 complex regulates loss of sister chromatid cohesion at the metaphase to anaphase transition in yeast. Cell 93: 1067-1076.

Flach, J., Bossie, M., Vogel, J., Corbett, A., Jinks, T., Willins, D.A., and Silver, P.A. 1994. A yeast RNA-binding protein shuttles between the nucleus and the cytoplasm. Mol. Cell. Biol. 14: 8399-8407.

Hilleren, P., McCarthy, T., Rosbash, M., Parker, R., and Jensen, T.H. 2001. Quality control of mRNA 3'-end processing is linked to the nuclear exosome. Nature 413: 538-542.

Jensen, T.H., Boulay, J., Rosbash, M., and Libri, D. 2001a. The DECD box putative ATPase Sub2p is an early mRNA export factor. Curr. Biol. 11: 1711-1715.

Jensen, T.H., Patricio, K., McCarthy, T., and Rosbash, M. 2001b. A block to mRNA nuclear export in S. cerevisiae leads to hyperadenylation of transcripts that accumulate at the site of transcription. Mol. Cell 7: 887-898.

Krebber, H., Taura, T., Lee, M.S., and Silver, P.A. 1999. Uncoupling of the hnRNP Npl3p from mRNAs during the stress-induced block in mRNA export. Genes \& Dev. 13: 1994-2004.

Lee, M.S., Henry, M., and Silver, P.A. 1996. A protein that shuttles between the nucleus and the cytoplasm is an important mediator of RNA export. Genes \& Dev. 10: 1233-1246.

Lei, E.P., Krebber, H., and Silver, P.A. 2001. Messenger RNAs are recruited for nuclear export during transcription. Genes \& Dev. 15: 1771-1782.

Libri, D., Dower, K., Boulay, J., Thomsen, R., Rosbash, M., and Jensen, T.H. 2002. Interactions between mRNA export commitment, 3'end quality control, and nuclear degradation. Mol. Cell. Biol. 22: 8254-8266.

Liu, Y., Liang, S., and Tartakoff, A.M. 1996. Heat shock disassembles the nucleolus and inhibits nuclear protein import and poly(A)+ RNA export. EMBO J. 15: 6750-6757.

Matera, A.G. 1999. Nuclear bodies: Multifaceted subdomains of the interchromatin space. Trends Cell Biol. 9: 302-309.

Reed, R. and Hurt, E. 2002. A conserved mRNA export machinery coupled to pre-mRNA splicing. Cell 108: 523-531.

Rout, M.P., Aitchison, J.D., Suprapto, A., Hjertaas, K., Zhao, Y., and Chait, B.T. 2000. The yeast nuclear pore complex: Composition, architecture, and transport mechanism. J. Cell Biol. 148: 635-651.

Samarsky, D.A., Fournier, M.J., Singer, R.H., and Bertrand, E. 1998. The snoRNA box C/D motif directs nucleolar targeting and also couples snoRNA synthesis and localization. EMBO J. 17: 37473757.

Strahm, Y., Fahrenkrog, B., Zenklusen, D., Rychner, E., Kantor, J., Rosbach, M., and Stutz, F. 1999. The RNA export factor Glelp is located on the cytoplasmic fibrils of the NPC and physically interacts with the FG-nucleoporin Riplp, the DEAD-box protein Rat8p/Dbp5p and a new protein Ymr 255p. EMBO J. 18: 57615777.

Strasser, K., Masuda, S., Mason, P., Pfannstiel, J., Oppizzi, M., Rodriguez-Navarro, S., Rondon, A.G., Aguilera, A., Struhl, K., Reed, R., et al. 2002. TREX is a conserved complex coupling transcription with messenger RNA export. Nature 417: 304-308.

Stutz, F., Kantor, J., Zhang, D., McCarthy, T., Neville, M., and Rosbash, M. 1997. The yeast nucleoporin riplp contributes to multiple export pathways with no essential role for its FG-repeat region. Genes \& Dev. 11: 2857-2868.

Vainberg, I.E., Dower, K., and Rosbash, M. 2000. Nuclear export of heat shock and non-heat-shock mRNA occurs via similar pathways. Mol. Cell. Biol. 20: 3996-4005.

Verheggen, C., Mouaikel, J., Thiry, M., Blanchard, J.M., Tollervey, D., Bordonne, R., Lafontaine, D.L., and Bertrand, E. 2001. Box C/D small nucleolar RNA trafficking involves small nucleolar RNP proteins, nucleolar factors and a novel nuclear domain. EMBO J. 20: 5480-5490.

Zenklusen, D., Vinciguerra, P., Wyss, J.C., and Stutz, F. 2002. Stable mRNP formation and export require cotranscriptional recruitment of the mRNA export factors Yralp and Sub2p by Hprlp. Mol. Cell. Biol. 22: 8241-8253. 

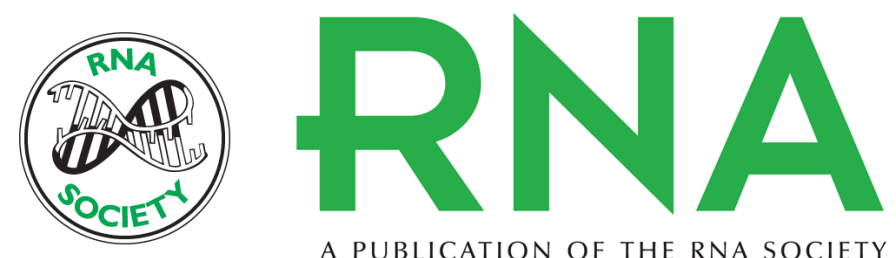

A PUBLICATION OF THE RNA SOCIETY

\section{Localization of nuclear retained mRNAs in Saccharomyces cerevisiae}

RUNE THOMSEN, DOMENICO LIBRI, JOCELYNE BOULAY, et al.

RNA 2003 9: 1049-1057

References This article cites 23 articles, 14 of which can be accessed free at:

http://rnajournal.cshlp.org/content/9/9/1049.full.html\#ref-list-1

License

Email Alerting Receive free email alerts when new articles cite this article - sign up in the box at the Service top right corner of the article or click here.

To subscribe to $R N A$ go to:

http://rnajournal.cshlp.org/subscriptions 